\title{
Asymptotic Analysis of Shout Options Close to Expiry
}

\author{
G. Alobaidi ${ }^{1}$ and R. Mallier ${ }^{2}$ \\ ${ }^{1}$ Department of Mathematics, American University of Sharjah, Sharjah, UAE \\ ${ }^{2}$ Department of Mathematics and Statistics, York University, Toronto, ON, Canada M3J 1P3 \\ Correspondence should be addressed to G. Alobaidi; galobaidi@aus.edu
}

Received 14 November 2013; Accepted 9 January 2014; Published 17 February 2014

Academic Editors: R. V. Roy and E. Skubalska-Rafajlowicz

Copyright (C) 2014 G. Alobaidi and R. Mallier. This is an open access article distributed under the Creative Commons Attribution License, which permits unrestricted use, distribution, and reproduction in any medium, provided the original work is properly cited.

We use an asymptotic expansion to study the behavior of shout options close to expiry. Series solutions are obtained for the location of the free boundary and the price of the option in that limit.

\section{Introduction}

Since the seminal work of Black and Scholes [1] and Merton [2] on the pricing of options appeared forty years ago, there has been a dramatic growth in both the role and complexity of financial contracts. The world's first organized options exchange, the Chicago Board of Options Exchange (CBOE), opened in 1973, the same year as $[1,2]$ appeared in print, and trading volumes for the standard options traded on exchanges such as the CBOE exploded in the late 1970's and early 1980's. Around the same time as the growth in standard options, financial institutions began to look for alternative forms of options, termed exotic options, both to meet their needs in terms of reallocating risk and also to increase their business. These exotics, which are usually traded over-the-counter (OTC), became very popular in the late 1980's and early 1990's, with their users including big corporations, financial institutions, fund managers, and private bankers.

One such exotic, which is the topic of the current study, is a shout option $[3,4]$. This option has the feature that it allows an investor to receive a portion of the pay-off prior to expiry while still retaining the right to profit from further upsides. In order to use this feature, the investor must shout, meaning exercise the option, at a time of his choosing, and this leads to an optimization problem wherein the investor must decide the best time at which to shout, which in turn leads to a free boundary problem, with the free boundary dividing the region where it is optimal to shout from that where it is not. In practice, shouting should of course only take place on the free boundary.
This sort of free boundary problem is of course common in the pricing of options with American-style early option features, and this aspect of vanilla American options has been studied extensively in, for example, the recent studies of [5-13], although American-style exotics have received somewhat less attention. In the present study, we will use a technique developed by Tao [14-22] for the free boundary problems arising in melting and solidification; such problems are termed Stefan problems. Tao used a series expansion in time to find the location of the moving surface of separation between two phases of a material, and, in almost all of the cases he studied, he found that the location of the interface was proportional to $\tau^{1 / 2}, \tau$ being the time from when the two phases were first put in contact. Although, like all equity options, shout options obey the Black-Scholes-Merton partial differential equation $[1,2]$, it is straightforward to use a change of variables $[8,23]$ to transform this into the heat conduction equation studied by Tao, along with a nonhomogeneous term, and, once this transformation has been made, it is straightforward to apply Tao's method. This approach has been taken for vanilla American options in the past $[5,6,8$, 12].

Before starting our analysis, which is presented in Section 2, we should first mention earlier work on shout options, much of which has been numerical, although as with other options involving a free boundary and choice on the part of an investor, some standard numerical techniques such as the forward-looking Monte Carlo method are problematic because of difficulties handling the optimization component 
of shout options. In [24], a Green's function approach was used. With this approach, it was assumed that early exercise could only occur on a limited number of fixed times $t<t_{1}<$ $t_{2}<\cdots<t_{n-1}<t_{n}=T$, so that the option was treated as Bermudan-style or semi-American rather than Americanstyle, and then the value of the option at time $t_{m}$ was used to compute the value at time $t_{m-1}$, which in turn was used to compute the value at time $t_{m-2}$ and so on. The value at time $t_{m-1}$ was computed by using an integral involving the product of the Green's function with the value at time $t_{m}$, with this integral being evaluated numerically. More standard numerical methods, such as finite differences, have also been applied to shout options [25]. One analytical study was [4] which used partial Laplace transforms to study the free boundary.

Finally in this section, we should mention that, in addition to shout options themselves, the shout feature can also be found embedded in several other financial contracts, some of which are offered to retail investors. One such contract is a segregated fund, sold by life insurance companies in Canada, which allows investors to lock in their profits prior to maturity. Some of these contracts have multiple shouting opportunities, although in this analysis, we assume that the holder can shout only once so that there is only one free boundary whose location must be optimized: with multiple shouting opportunities, there would be multiple free boundaries.

\section{Analysis}

As with any equity option, the price $V(S, t)$ of a shout option is governed by the Black-Scholes-Merton partial differential equation $(\mathrm{PDE})[1,2]$

$$
\frac{\partial V}{\partial t}+\frac{\sigma^{2} S^{2}}{2} \frac{\partial^{2} V}{\partial S^{2}}+(r-D) S \frac{\partial V}{\partial S}-r V=0
$$

where $S$ is the price of the underlying and $t<T$ is the time, with $T$ being the expiry time. The parameters in this equation are the risk-free rate, $r$, the dividend yield, $D$, and the volatility, $\sigma$, all of which are assumed constant here. Merton [26] observed that this same PDE (1) governs the price of many different securities, and it is the boundary and initial conditions which differentiate the securities, not the PDE. For the shout options considered here, the pay-off for an option held to maturity without shouting is $\max (S-E, 0)$ for a call and $\max (E-S, 0)$ for a put, where $E$ is the original strike price of the shout option; these pay-offs are the same as for vanilla European and American options. In addition, a shout option gives the holder the right to cash in some of the gains prior to expiry, and a shout call can be exchanged at any time for the excess of the current stock price $S$ over the strike price $E$ together with a European call with a new strike price equal to the current stock price, provided the stock price is greater than the original exercise price. Obviously, the price of such a European call can be written down using the Black-Scholes option pricing formula, which means that, upon shouting, the holder of a call receives a package consisting of cash together with a European call with a total value of

$$
\begin{aligned}
V_{f}(S, \tau)= & S-E+\frac{S e^{-D \tau}}{2} \operatorname{erfc}\left[-\frac{\left(r-D+\sigma^{2} / 2\right) \sqrt{\tau}}{\sqrt{2} \sigma}\right] \\
& -\frac{S e^{-r \tau}}{2} \operatorname{erfc}\left[-\frac{\left(r-D-\sigma^{2} / 2\right) \sqrt{\tau}}{\sqrt{2} \sigma}\right] .
\end{aligned}
$$

Obviously, this leads to the constraint that the value of a shout option cannot be less than the proceeds from shouting immediately, so that, for the call, $V \geq V_{f}$ for $S \geq E$, where $V_{f}$ is the pay-off from shouting. Similarly, a shout put can be exchanged at any time for the deficit of the current stock price $S$ below the strike price $E$ together with a European put with a new strike price equal to the current stock price, provided the stock price is less than the original exercise price. Upon shouting, therefore, the holder of a put receives a package consisting of cash together with a European put with a total value of

$$
\begin{aligned}
V_{f}(S, \tau)=E & -S-\frac{S}{2} e^{-D \tau} \operatorname{erfc}\left[\frac{\left(r-D+\sigma^{2} / 2\right) \sqrt{\tau}}{\sqrt{2} \sigma}\right] \\
& +\frac{S}{2} e^{-r \tau} \operatorname{erfc}\left[\frac{\left(r-D-\sigma^{2} / 2\right) \sqrt{\tau}}{\sqrt{2} \sigma}\right],
\end{aligned}
$$

and for a put we have the constraint that $V \geq V_{f}$ for $S \leq$ $E$. In both (2) and (3), erfc denotes the complementary error function.

As with American options, the possibility of "shouting" leads to a free boundary where it is optimal to shout. Several properties of this free boundary are known. Firstly, we know the value of the option at the free boundary, namely, $V_{f}$, given by (2) and (3) above, and also the value of the option's delta, or derivative of its value with respect to the stock price, at the free boundary, where it is equal to $\left(\partial V_{f} / \partial S\right)$, which for a call is

$$
\frac{V_{f}(S, \tau)+E}{S}
$$

while for a put it is

$$
\frac{V_{f}(S, \tau)-E}{S} .
$$

The condition on the delta, $(\partial V / \partial S)$, comes from requiring that the delta is continuous across the boundary and is essentially the "high contact" or "smooth-pasting" condition, which was first proposed by Samuelson [27] for American options.

Secondly, we know that the location of the free boundary at expiry $\tau=0$ is $S_{f}(0)=E$, which can be deduced intuitively because the pay-off for early exercise is so sweet for shout options. In our terms, $\tau_{f}(E)=0$. We also know that the optimal exercise boundary moves upwards (or at worst is 
flat) as we move away from the expiration date for a call and downwards (or again at worst is flat) for a put.

To analyze this equation, we will use an expansion which is essentially along the lines of those used by Tao [14-22]. An approach very similar to this has previously been applied to American options $[5,6,8,12]$. To apply Tao's method to the Black-Scholes-Merton PDE (1), it is necessary to make a change of variables to transform (1) into a more standard diffusion equation together with a forcing term. We will proceed along the same lines as $[5,6,8,12]$ and make the change of variables $S=E e^{x}, t=T-2 \tau / \sigma^{2}$, and $V(S, t)=V_{f}+E v(x, \tau)$, which leads us to the diffusion-like PDE

$$
\frac{\partial v}{\partial \tau}=\frac{\partial^{2} v}{\partial x^{2}}+k_{2} \frac{\partial v}{\partial x}-k_{1} v+f(x, \tau)
$$

where $k_{1}=2 r / \sigma^{2}$ and $k_{2}=2(r-D) / \sigma^{2}-1$ and the nonhomogeneous term for the call is

$$
\begin{aligned}
f(x, \tau)= & k_{1}+\left(1+k_{2}-k_{1}\right) e^{x} \\
& -\frac{e^{x-k_{1} \tau}}{2} \\
& \times\left(\frac{e^{-k_{2}^{2} \tau / 4}}{\sqrt{\pi \tau}}+\left(k_{2}+1\right) \operatorname{erfc}\left[-\frac{k_{2} \sqrt{\tau}}{2}\right]\right),
\end{aligned}
$$

while for the put it is

$$
\begin{aligned}
f(x, \tau)= & -k_{1}-\left(1+k_{2}-k_{1}\right) e^{x} \\
& -\frac{e^{x-k_{1} \tau}}{2} \\
& \times\left(\frac{e^{-k_{2}^{2} \tau / 4}}{\sqrt{\pi \tau}}-\left(k_{2}+1\right) \operatorname{erfc}\left[\frac{k_{2} \sqrt{\tau}}{2}\right]\right) .
\end{aligned}
$$

Equation (6) is valid for $\tau>0$ and must be solved together with the payoff at expiry, $\tau=0$, which is $v(x, 0)=\max (1-$ $\left.e^{x}, 0\right)$ for a call and $v(x, 0)=\max \left(e^{x}-1,0\right)$ for a put, while on the free boundary, we have

$$
v=\frac{\partial v}{\partial x}=0
$$

At expiry the free boundary starts at $S=E$ or equivalently $x=0$. In the analysis that follows, strictly speaking (6) is valid only where it is valid to hold the option, so that at expiry, we can only impose the initial condition on $x \leq 0$ for the call and on $x \geq 0$ for the put.

To tackle (6) and associated boundary and initial conditions, we will follow Tao [14-22] and seek a series solution of the form

$$
v(x, \tau)=\sum_{n=1}^{\infty} \tau^{n / 2} V_{n}(\xi),
$$

where $\xi=x / 2 \sqrt{\tau}$ is a similarity variable, while we assume that the free boundary is located at $x=x_{f}(\tau)$ which we also write as a series as follows:

$$
x_{f}(\tau)=\sum_{n=1}^{\infty} x_{n} \tau^{n / 2} .
$$

In our analysis, we substitute the assumed form for $v(x, \tau)$ (10) in the PDE (6) and group powers of $\tau$. To abbreviate the presentation, we introduce the operator

$$
L_{n} \equiv \frac{1}{4} \frac{d^{2}}{d \xi^{2}}+\frac{1}{2} \frac{d}{d \xi}-\frac{n}{2}
$$

2.1. The Call. For the call, at the first few orders, we find the following equations for the various $V_{n}$ :

$$
\begin{gathered}
L_{1} V_{1}=\frac{1}{2 \sqrt{\pi}}, \\
L_{2} V_{2}=-\frac{k_{2}}{2} V_{1}^{\prime}-\frac{k_{2}+1}{2}+\frac{\xi}{\sqrt{\pi}}, \\
L_{3} V_{3}=-\frac{k_{2}}{2} V_{2}^{\prime}-k_{1} V_{1}+\left(2 k_{1}-k_{2}-1\right) \xi \\
+\frac{\xi^{2}-\left(k_{1} / 2\right)+\left(3 k_{2}^{2} / 8\right)+\left(k_{2} / 2\right)}{\sqrt{\pi}}, \\
L_{4} V_{4}=-\frac{k_{2}}{2} V_{3}^{\prime}-k_{1} V_{2}+\left(2 k_{1}-1-k_{2}\right) \xi^{2} \\
-\frac{1}{2} k_{1}\left(k_{2}+1\right) \\
+\frac{\left(2 \xi^{3} / 3\right)+\left(k_{2}-k_{1}+\left(3 k_{2}^{2} / 4\right)\right) \xi}{\sqrt{\pi}},
\end{gathered}
$$

with similar equations for the higher orders, and it is straightforward to write the solutions to (13) which satisfy the initial condition that $v(x, 0)=\max \left(1-e^{x}, 0\right)$ for $x \leq 0$ or equivalently $V_{n} \rightarrow-(2 \xi)^{n} / n !$ as $\xi \rightarrow-\infty$. The solutions for the first few orders are

$$
\begin{gathered}
V_{1}=-2 \xi-\frac{1}{\sqrt{\pi}}+C_{1}\left[\frac{e^{-\xi^{2}}}{\sqrt{\pi}}+\xi \operatorname{erfc}(-\xi)\right], \\
V_{2}=-2 \xi^{2}-\frac{2 \xi}{\sqrt{\pi}}-\frac{k_{2}+1}{2} \\
+C_{2}\left[\frac{2 \xi e^{-\xi^{2}}}{\sqrt{\pi}}+\left(2 \xi^{2}+1\right) \operatorname{erfc}(-\xi)\right] \\
\quad-C_{1} k_{2}\left[\frac{\xi e^{-\xi^{2}}}{\sqrt{\pi}}+\xi^{2} \operatorname{erfc}(-\xi)\right], \\
V_{3}=\frac{-8+12 k_{1}-12 k_{2}-3 k_{2}^{2}-24 \xi^{2}}{12 \sqrt{\pi}} \\
+\frac{\xi}{3}\left(4 \xi^{2}+3 k_{2}+3\right) \\
+C_{3}\left[\left(1+\xi^{2}\right) \frac{e^{-\xi^{2}}}{\sqrt{\pi}}+\left(\xi^{3}+\frac{3 \xi}{2}\right) \operatorname{erfc}(-\xi)\right]
\end{gathered}
$$




$$
\begin{aligned}
&+ 2 C_{2} k_{2}\left[\frac{e^{-\xi^{2}}}{\sqrt{\pi}}+\xi \operatorname{erfc}(-\xi)\right] \\
&- C_{1}\left[\left(k_{1}+\frac{3}{4} k_{2}^{2}\right) \frac{e^{-\xi^{2}}}{\sqrt{\pi}}+\xi\left(k_{1}+k_{2}^{2}\right) \operatorname{erfc}(-\xi)\right], \\
& V_{4}=\frac{\xi\left(-8 \xi^{2}+12 k_{1}-12 k_{2}-8-3 k_{2}^{3}\right)}{6 \sqrt{\pi}} \\
&-\frac{2 \xi^{4}}{3}-\left(k_{2}+1\right) \xi^{2}-\frac{\left(k_{2}+1\right)^{2}}{4}+\frac{k_{1}\left(k_{2}+1\right)}{2} \\
&+C_{4}\left[\frac{\left(5 \xi+2 \xi^{3}\right) e^{-\xi^{2}}}{3 \sqrt{\pi}}+\left(\frac{4}{3} \xi^{4}+4 \xi^{2}+1\right) \operatorname{erfc}(-\xi)\right] \\
&+C_{3} k_{2}\left[\frac{\left(\xi+\xi^{3}\right) e^{-\xi^{2}}}{\sqrt{\pi}}+\left(\frac{3}{2} \xi^{4}+\xi^{2}\right) \operatorname{erfc}(-\xi)\right] \\
&+C_{2}\left[\frac{\left(\left(4 k_{1}-5 k_{2}^{2}\right) \xi+\left(4 k_{1}-2 k_{2}^{2}\right) \xi^{3}\right) e^{-\xi^{2}}}{3 \sqrt{\pi}}\right. \\
&+\left(\frac{2}{3}\left(2 k_{1}-k_{2}^{2}\right) \xi^{4}+2\left(k_{1}-k_{2}^{2} \xi^{2}\right)\right) \\
&+C_{1} k_{2}\left[\left(\left(\frac{3}{4} k_{2}^{2}+1\right) \xi+\frac{k_{2}^{2} \xi^{3}}{3}\right) \frac{e^{-\xi^{2}}}{\sqrt{\pi}}\right. \\
&\times \operatorname{erfc}(-\xi)] \\
&\left.\left.+\left(k_{2}^{2}+k_{1}\right) \xi^{2}+\frac{k_{2}^{2} \xi^{4}}{3}\right) \operatorname{erfc}(-\xi)\right], \\
&+
\end{aligned}
$$

where the $C_{n}$ are constants that must be found by applying the conditions (9) at the free boundary.

To apply the conditions (9) at the free boundary, we reconstitute the series (10) using the expressions (14) for the $V_{n}$ and then substitute the assumed form (11) for $x_{f}(\tau)$ and again group powers of $\tau$. The solution of the resulting equations will yield the coefficients $x_{n}$ and $C_{n}$.

Proceeding in this manner, at leading order, we obtain the pair of equations for $x_{1}$ and $C_{1}$ :

$$
\begin{gathered}
C_{1}\left[\frac{e^{-x_{1}^{2} / 4}}{\sqrt{\pi}}+\frac{x_{1}}{2} \operatorname{erfc}\left(-\frac{x_{1}}{2}\right)\right]-x_{1}-\frac{1}{\sqrt{\pi}}=0, \\
\frac{C_{1}}{2} \operatorname{erfc}\left(-\frac{x_{1}}{2}\right)-1=0,
\end{gathered}
$$

so that $x_{1}$ must satisfy the equation

$$
\operatorname{erfc}\left(-\frac{x_{1}}{2}\right)=2 e^{-x_{1}^{2} / 4}
$$

with $C_{1}$ then given by

$$
C_{1}=\frac{2}{\operatorname{erfc}\left(-x_{1} / 2\right)}=e^{x_{1}^{2} / 4} .
$$

These expressions ((16) and (17)) are similar to but not identical to their counterparts for the American call with $r>D$ given in $[5,8]$; the analysis of the American call with $r \leq D$ is rather different and involves logarithms. As with $[5,8],(16)$ and (17) must be solved numerically, and we find

$$
\begin{aligned}
& x_{1}=1.030396155, \\
& C_{1}=1.303990345 .
\end{aligned}
$$

At the next order on the free boundary, we obtain the pair of equations

$$
\begin{gathered}
\frac{C_{2}}{C_{1}}\left[2+\frac{x_{1}}{\sqrt{\pi}}+x_{1}^{2}\right]-\frac{k_{2}}{2}\left[1+\frac{x_{1}}{\sqrt{\pi}}+x_{1}^{2}\right] \\
-\frac{1}{2}\left[1+\frac{2 x_{1}}{\sqrt{\pi}}+x_{1}^{2}\right]=0, \\
\frac{C_{2}}{C_{1}}\left[4 x_{1}+\frac{1}{\sqrt{\pi}}\right]-k_{2}\left[2 x_{1}+\frac{1}{\sqrt{\pi}}\right]-\left[2 x_{1}+\frac{2-x_{2}}{\sqrt{\pi}}\right]=0,
\end{gathered}
$$

which have a solution

$$
\begin{aligned}
x_{2} & =\frac{2 \pi x_{1}\left(1+k_{2}\right)+\sqrt{\pi}\left(2-x_{1}^{2}\left(k_{2}+2\right)\right)-x_{1}\left(k_{2}+2\right)}{\sqrt{\pi}\left(2+x_{1}^{2}\right)+x_{1}} \\
& =0.5516261066 k_{2}+0.6496056829, \\
C_{2} & =\frac{C_{1}}{2}\left[\frac{\sqrt{\pi}\left(1+k_{2}\right)\left(1+x_{1}^{2}\right)+x_{1}\left(2+k_{2}\right)}{\sqrt{\pi}\left(2+x_{1}^{2}\right)+x_{1}}\right] \\
& =0.4730258268 k_{2}+0.5770676475 .
\end{aligned}
$$

If we continue the analysis to higher orders, it is straightforward to show that

$$
\begin{aligned}
x_{3}= & 0.292207785 k_{2}^{2}+0.893808684 k_{2} \\
& -0.3950836719 k_{1}+0.610198033, \\
x_{4}= & 0.203979819 k_{2}^{3}+0.9698633439 k_{2}^{2} \\
& +1.452965717 k_{2}-0.8345732085 k_{1} k_{2} \\
& -0.9203954739 k_{1}+0.6875373101, \\
x_{5}= & 0.1638998310 k_{2}^{4}+1.037819212 k_{2}^{3} \\
& +2.400660126 k_{2}^{2}+2.386097546 k_{2} \\
& -1.469136768 k_{1} k_{2}^{2}-3.178694724 k_{1} k_{2} \\
& -1.768309502 k_{1}+0.4855736529 k_{1}^{2} \\
& +0.8593396200,
\end{aligned}
$$


with

$$
\begin{aligned}
C_{3}= & 0.2474229510 k_{2}^{2}-0.1188463797 k_{2} \\
& +0.5304417776 k_{1}+0.7375354641 \\
C_{4}= & 0.00318404397 k_{2}^{3}+0.2473130714 k_{2}^{2} \\
& +0.5280891544 k_{2}-0.3593239715 k_{1} k_{2} \\
& -0.4660110242 k_{1}+0.2730318578 \\
C_{5}= & 0.04939807173 k_{2}^{4}-0.02503076966 k_{2}^{3} \\
& +0.1638363804 k_{2}^{2}-0.00645471784 k_{2} \\
& +0.1970541793 k_{1} k_{2}^{2}-0.08183232660 k_{1} k_{2} \\
& +0.03734886087 k_{1}+0.09802891395 k_{1}^{2} \\
& +0.1461511036 .
\end{aligned}
$$

In (10), (14), (18), (20), and (22), we have an expression for the value of a shout call close to expiry, with the location of the free boundary given by (11), (18), (20), and (21).

2.2. The Put. The analysis for the put is very similar to that for the call, but with a different nonhomogeneous term and different initial condition. Once again using the operator $L_{n}$ defined in (12), at the first few orders, we find

$$
\begin{gathered}
L_{1} V_{1}=\frac{1}{2 \sqrt{\pi}}, \\
L_{2} V_{2}=\frac{k_{2}}{2} V_{1}^{\prime}+\frac{k_{2}+1}{2}+\frac{\xi}{\sqrt{\pi}}, \\
L_{3} V_{3}=-\frac{k_{2}}{2} V_{2}^{\prime}-k_{1} V_{1}+\left(k_{2}+1-2 k_{1}\right) \xi \\
+\frac{\xi^{2}-\left(k_{1} / 2\right)+\left(3 k_{2}^{2} / 8\right)+\left(k_{2} / 2\right)}{\sqrt{\pi}}, \\
L_{4} V_{4}=-\frac{k_{2}}{2} V_{3}^{\prime}-k_{1} V_{2}+\left(k_{2}+1-2 k_{1}\right) \xi^{2} \\
+\frac{1}{2} k_{1}\left(k_{2}+1\right)+\frac{\left(k_{2}-k_{1}+\left(3 k_{2}^{2} / 4\right)\right) \xi+\left(2 \xi^{3} / 3\right)}{\sqrt{\pi}} .
\end{gathered}
$$

Not surprisingly, (23) for the put are very similar to those (13) for the call, differing only in the signs of various nonhomogeneous terms. Once again, it is straightforward to write the solutions to (23) which satisfy the initial condition, which for the put is $v(x, 0)=\max \left(e^{x}-1,0\right)$ for $x \geq 0$ or equivalently $V_{n} \rightarrow(2 \xi)^{n} / n$ ! as $\xi \rightarrow+\infty$. The solutions for the first few orders are

$$
V_{1}=2 \xi-\frac{1}{\sqrt{\pi}}+C_{1}\left[\frac{e^{-\xi^{2}}}{\sqrt{\pi}}-\xi \operatorname{erfc}(\xi)\right],
$$

$$
\begin{aligned}
& V_{2}=2 \xi^{2}-\frac{2 \xi}{\sqrt{\pi}}+\frac{k_{2}+1}{2} \\
& -C_{2}\left[\frac{2 \xi e^{-\xi^{2}}}{\sqrt{\pi}}-\left(2 \xi^{2}+1\right) \operatorname{erfc}(\xi)\right] \\
& -C_{1} k_{2}\left[\frac{\xi e^{-\xi^{2}}}{\sqrt{\pi}}-\xi^{2} \operatorname{erfc}(\xi)\right] \text {, } \\
& V_{3}=\frac{-8+12 k_{1}-12 k_{2}-3 k_{2}^{2}-24 \xi^{2}}{12 \sqrt{\pi}} \\
& +\frac{\xi}{3}\left(4 \xi^{2}+3 k_{2}+3\right) \\
& -C_{3}\left[\left(1+\xi^{2}\right) \frac{e^{-\xi^{2}}}{\sqrt{\pi}}-\left(\xi^{3}+\frac{3 \xi}{2}\right) \operatorname{erfc}(\xi)\right] \\
& -2 C_{2} k_{2}\left[\frac{e^{-\xi^{2}}}{\sqrt{\pi}}-\xi \operatorname{erfc}(\xi)\right] \\
& -C_{1}\left[\left(k_{1}+\frac{3}{4} k_{2}^{2}\right) \frac{e^{-\xi^{2}}}{\sqrt{\pi}}-\xi\left(k_{1}+k_{2}^{2}\right) \operatorname{erfc}(\xi)\right] \text {, } \\
& V_{4}=\frac{\xi\left(-8 \xi^{2}+12 k_{1}-12 k_{2}-8-3 k_{2}^{3}\right)}{6 \sqrt{\pi}} \\
& +\frac{2 \xi^{4}}{3}+\left(k_{2}+1\right) \xi^{2}+\frac{\left(k_{2}+1\right)^{2}}{4}-\frac{k_{1}\left(k_{2}+1\right)}{2} \\
& -C_{4}\left[\frac{\left(5 \xi+2 \xi^{3}\right) e^{-\xi^{2}}}{3 \sqrt{\pi}}-\left(\frac{4}{3} \xi^{4}+4 \xi^{2}+1\right) \operatorname{erfc}(\xi)\right] \\
& -C_{3} k_{2}\left[\frac{\left(\xi+\xi^{3}\right) e^{-\xi^{2}}}{\sqrt{\pi}}-\left(\frac{3}{2} \xi^{4}+\xi^{2}\right) \operatorname{erfc}(\xi)\right] \\
& -C_{2}\left[\frac{\left(\left(4 k_{1}-5 k_{2}^{2}\right) \xi+\left(4 k_{1}-2 k_{2}^{2}\right) \xi^{3}\right) e^{-\xi^{2}}}{3 \sqrt{\pi}}\right. \\
& \left.-\left(\frac{2}{3}\left(2 k_{1}-k_{2}^{2}\right) \xi^{4}+2\left(k_{1}-k_{2}^{2} \xi^{2}\right)\right) \operatorname{erfc}(\xi)\right] \\
& -C_{1} k_{2}\left[\left(\left(\frac{3}{4} k_{2}^{2}+1\right) \xi+\frac{k_{2}^{2} \xi^{3}}{3}\right) \frac{e^{-\xi^{2}}}{\sqrt{\pi}}\right. \\
& \left.-\left(\left(k_{2}^{2}+k_{1}\right) \xi^{2}+\frac{k_{2}^{2} \xi^{4}}{3}\right) \operatorname{erfc}(\xi)\right],
\end{aligned}
$$

which differ from their counterparts (14) for the call only in the signs of various terms. 
To apply the conditions (9) at the free boundary, we proceed as for the call, and at leading order, we obtain the pair of equations for $x_{1}$ and $C_{1}$,

$$
\begin{gathered}
C_{1}\left[\frac{e^{-x_{1}^{2} / 4}}{\sqrt{\pi}}-\frac{x_{1}}{2} \operatorname{erfc}\left(\frac{x_{1}}{2}\right)\right]+x_{1}-\frac{1}{\sqrt{\pi}}=0, \\
\frac{C_{1}}{2} \operatorname{erfc}\left(\frac{x_{1}}{2}\right)-1=0,
\end{gathered}
$$

so that $x_{1}$ obeys

$$
\operatorname{erfc}\left(\frac{x_{1}}{2}\right)=2 e^{-x_{1}^{2} / 4},
$$

with $C_{1}$ given by

$$
C_{1}=\frac{2}{\operatorname{erfc}\left(x_{1} / 2\right)}=e^{-x_{1}^{2} / 4},
$$

which can be solved numerically to give

$$
\begin{aligned}
& x_{1}=-1.030396155, \\
& C_{1}=1.303990345 .
\end{aligned}
$$

The coefficient $C_{1}$ is the same as for the call but the sign of $x_{1}$ is changed.

At the next order, we have

$$
\begin{gathered}
\frac{C_{2}}{C_{1}}\left[2-\frac{x_{1}}{\sqrt{\pi}}+x_{1}^{2}\right]+\frac{k_{2}}{2}\left[1-\frac{x_{1}}{\sqrt{\pi}}+x_{1}^{2}\right] \\
+\frac{1}{2}\left[1-\frac{2 x_{1}}{\sqrt{\pi}}+x_{1}^{2}\right]=0, \\
\frac{C_{2}}{C_{1}}\left[4 x_{1}-\frac{1}{\sqrt{\pi}}\right]+k_{2}\left[2 x_{1}-\frac{1}{\sqrt{\pi}}\right]+\left[2 x_{1}+\frac{x_{2}-2}{\sqrt{\pi}}\right]=0,
\end{gathered}
$$

with a solution

$$
\begin{aligned}
x_{2} & =\frac{-2 \pi x_{1}\left(1+k_{2}\right)+\sqrt{\pi}\left(2-x_{1}^{2}\left(k_{2}+2\right)\right)-x_{1}\left(k_{2}+2\right)}{\sqrt{\pi}\left(2+x_{1}^{2}\right)-x_{1}} \\
& =0.5516261066 k_{2}+0.6496056829, \\
C_{2} & =\frac{C_{1}}{2}\left[-\frac{\sqrt{\pi}\left(1+k_{2}\right)\left(1+x_{1}^{2}\right)+x_{1}\left(2+k_{2}\right)}{\sqrt{\pi}\left(2+x_{1}^{2}\right)-x_{1}}\right] \\
& =-0.4730258268 k_{2}-0.5770676475,
\end{aligned}
$$

with $x_{2}$ the same as for the call but the sign of $C_{2}$ reversed. For the higher orders, we find

$$
\begin{aligned}
x_{3}= & -0.292207785 k_{2}^{2}+0.893808684 k_{2} \\
& -0.3950836719 k_{1}-0.610198033, \\
x_{4}= & 0.203979819 k_{2}^{3}+0.9698633439 k_{2}^{2} \\
& +1.452965717 k_{2}-0.8345732085 k_{1} k_{2} \\
& -0.9203954739 k_{1}+0.6875373101, \\
x_{5}= & -0.1638998310 k_{2}^{4}-1.037819212 k_{2}^{3} \\
& -2.400660126 k_{2}^{2}-2.386097546 k_{2} \\
& +1.469136768 k_{1} k_{2}^{2}+3.178694724 k_{1} k_{2} \\
& +1.768309502 k_{1}-0.4855736529 k_{1}^{2} \\
& -0.8593396200,
\end{aligned}
$$

with

$$
\begin{aligned}
C_{3}= & -0.2474229510 k_{2}^{2}+0.1188463797 k_{2} \\
& -0.5304417776 k_{1}-0.737535464, \\
C_{4}= & -0.00318404397 k_{2}^{3}+0.2473130714 k_{2}^{2} \\
& -0.5280891544 k_{2}+0.3593239715 k_{1} k_{2} \\
& +0.4660110242 k_{1}-0.2730318578, \\
C_{5}= & -0.04939807173 k_{2}^{4}+0.02503076966 k_{2}^{3} \\
& -0.1638363804 k_{2}^{2}+0.00645471784 k_{2} \\
& -0.1970541793 k_{1} k_{2}^{2}+0.08183232660 k_{1} k_{2} \\
& -0.03734886087 k_{1}-0.09802891395 k_{1}^{2} \\
& -0.1461511036,
\end{aligned}
$$

with (31) and (32) differing from their counterparts for the call ((21), (22)) only in the sign of various terms. In (10), (24), (28), (30), and (32), we have an expression for the value of a shout put close to expiry, with the location of the free boundary given by (11), (28), (30), and (31).

\section{Discussion}

In the previous section, we used the method of Tao [14-22] to study the behavior of shout options close to expiry, these being exotic options which allow the investor to receive a portion of the pay-off prior to expiry while still retaining the right to further upside participation, because of which the pay-off for early exercise is sweeter than for vanilla American options. Perhaps surprisingly, the behavior close to expiry is slightly different for shouts than for vanilla Americans, and we can attribute a large part of this difference to the richness of the pay-off for early exercise. For vanilla Americans [5-13], 
the behavior of the free boundary has a strong dependence on the relative values of the risk-free interest rate $r$ and the dividend yield $D$ on the underlying stock. For the American call with $0 \leq D<r$ and the American put with $D>r \geq 0$, the free boundary started at $r E / D$ at expiry, with $E$ as the exercise price of the option, and had the usual $\tau^{1 / 2}$ behavior close to expiry, meaning that, as $t \rightarrow T$, the free boundary behaved like $S \sim S_{0} \exp \left[a(T-t)^{1 / 2}\right]$; this was the $\tau^{1 / 2}$ behavior which Tao found in the majority of the physical problems he considered. For the American call with $D>r \geq 0$ and the American put with $0 \leq D<r$, the free boundary started at $E$ at expiry and behaved like $S \sim S_{0} \exp \left[a(-(T-t) \ln (T-t))^{1 / 2}\right]$; this behavior is somewhat unusual in that Tao did not encounter this sort of behavior in his studies. For shouts, we found in Section 2 that, although the coefficients in the expansion depended on $r$ and $D$, the qualitative behavior of the free boundary did not: regardless of the values of $r$ and $D$, the free boundary for a shout always starts from $E$ at expiry and always has the usual $\tau^{1 / 2}$ behavior close to expiry. Regardless of the values of $r$ and $D$, the free boundary close to expiry for shout options seems to be less steep than that for vanilla Americans, and it would seem likely that this is because early exercise is more likely for a shout than a vanilla American on the same underlying with the same strike, simply because the rewards for early exercise are greater for a shout than an American. For an American, early exercise involves a trade-off between receiving the pay-off earlier and receiving benefits from any further upside, while with a shout early exercise results in receiving a portion of the pay-off earlier while still benefitting from further upsides. Because of this, it would appear paradoxically that, although shout options are more complex contracts than vanilla Americans, the analysis of shouts is actually a little simpler than that of Americans, primarily because logs are not present for the shouts.

Finally, we note that the behavior of shout puts and calls close to expiry is very similar, suggesting that there is some sort of put-call symmetry for shout options, perhaps along the lines of that for vanilla Americans [28, 29], and it would be interesting to find the exact forms of this symmetry for shouts and other American-style exotics.

\section{Conflict of Interests}

The authors declare that there is no conflict of interests regarding the publication of this paper.

\section{References}

[1] F. Black and M. Scholes, "The pricing of options and corporate liabilities," Journal of Political Economy, vol. 81, pp. 637-659, 1973.

[2] R. C. Merton, "Theory of rational option pricing," The Bell Journal of Economics and Management Science, vol. 4, pp. 141183, 1973.

[3] B. Thomas, "Something to shout about," Risk, vol. 6, pp. 56-58, 1993.

[4] G. Alobaidi, R. Mallier, and S. Mansi, "Laplace transforms and shout options," Acta Mathematica Universitatis Comenianae, vol. 80, no. 1, pp. 79-102, 2011.
[5] G. Alobaidi and R. Mallier, "Asymptotic analysis of American call options," International Journal of Mathematics and Mathematical Sciences, vol. 27, no. 3, pp. 177-188, 2001.

[6] G. Alobaidi and R. Mallier, "On the optimal exercise boundary for an American put option," Journal of Applied Mathematics, vol. 1, no. 1, pp. 39-45, 2001.

[7] G. Barles, J. Burdeau, M. Romano, and M. Samsoen, "Critical stock price near expiration," Mathematical Finance, vol. 5, no. 2, pp. 77-95, 1995.

[8] J. N. Dewynne, S. D. Howison, I. Rupf, and P. Wilmott, "Some mathematical results in the pricing of American options," European Journal of Applied Mathematics, vol. 4, no. 4, pp. 381398, 1993.

[9] J. D. Evans, R. Kuske, and J. B. Keller, "American options of assets with dividends near expiry," Mathematical Finance, vol. 12, no. 3, pp. 219-237, 2002.

[10] C. Knessl, "A note on a moving boundary problem arising in the American put option," Studies in Applied Mathematics, vol. 107, no. 2, pp. 157-183, 2001.

[11] R. E. Kuske and J. B. Keller, "Optimal exercise boundary for an American put option," Applied Mathematical Finance, vol. 5, no. 2, pp. 107-116, 1998.

[12] R. Mallier and G. Alobaidi, "The American put option close to expiry," Acta Mathematica Universitatis Comenianae, vol. 73, no. 2, pp. 161-174, 2004.

[13] R. Stamicar, D. Ševčovič, and J. Chadam, "The early exercise boundary for the American put near expiry: numerical approximation," The Canadian Applied Mathematics Quarterly, vol. 7, no. 4, pp. 427-444, 1999.

[14] L. N. Tao, "The Stefan problem with arbitrary initial and boundary conditions," Quarterly of Applied Mathematics, vol. 36, no. 3, pp. 223-233, 1978.

[15] L. N. Tao, "Free boundary problems with radiation boundary conditions," Quarterly of Applied Mathematics, vol. 37, no. 1, pp. $1-10,1979$.

[16] L. N. Tao, "On solidification problems including the density jump at the moving boundary," The Quarterly Journal of Mechanics and Applied Mathematics, vol. 32, no. 2, pp. 175-185, 1979.

[17] L. N. Tao, "On free boundary problems with arbitrary initial and flux conditions," Zeitschrift für Angewandte Mathematik und Physik, vol. 30, no. 3, pp. 416-426, 1979.

[18] L. N. Tao, "The analyticity of solutions of the Stefan problem," Archive for Rational Mechanics and Analysis, vol. 72, no. 3, pp. 285-301, 1980.

[19] L. N. Tao, "On solidification of a binary alloy," The Quarterly Journal of Mechanics and Applied Mathematics, vol. 33, no. 2, pp. 211-225, 1980.

[20] L. N. Tao, "The exact solutions of some Stefan problems with prescribed heat flux," Journal of Applied Mechanics, vol. 48, no. 4, pp. 732-736, 1981.

[21] L. N. Tao, "The Stefan problem with an imperfect thermal contact at the interface," Journal of Applied Mechanics, vol. 49, no. 4, pp. 715-720, 1982.

[22] L. N. Tao, “The Cauchy-Stefan problem," Acta Mechanica, vol. 45, no. 1-2, pp. 49-64, 1982.

[23] P. Wilmott, Paul Wilmott on Quantitative Finance, John Wiley \& Sons, Chichester, UK, 2000.

[24] P. P. Boyle, A. W. Kolkiewicz, and K. S. Tan, "Valuation of the reset option in segregated fund contracts using quasiMonte Carlo," Research Report 99-10, Institute of Insurance and Pension Research, University of Waterloo, 1999. 
[25] H. Windcliff, P. A. Forsyth, and K. R. Vetzal, "Shout options: a framework for pricing contracts which can be modified by the investor," Journal of Computational and Applied Mathematics, vol. 134, no. 1-2, pp. 213-241, 2001.

[26] R. C. Merton, "On the problem of corporate debt: the risk structure of interest rates," The Journal of Finance, vol. 29, pp. 449-470, 1974.

[27] P. A. Samuelson, "Rational theory of warrant pricing," Industrial Management Review, vol. 6, pp. 13-31, 1965.

[28] M. Chesney and R. Gibson, "State space symmetry and two factor option pricing models," Advances in Futures and Operations Research, vol. 8, pp. 85-112, 1993.

[29] R. McDonald and M. Schroder, "A parity result for American options," Journal of Computational Finance, vol. 1, pp. 5-13, 1998. 


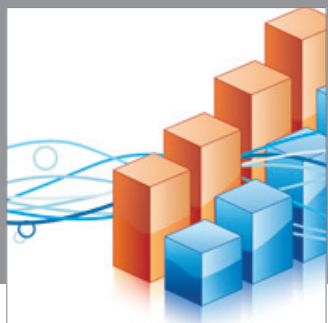

Advances in

Operations Research

mansans

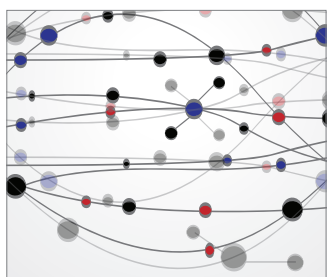

The Scientific World Journal
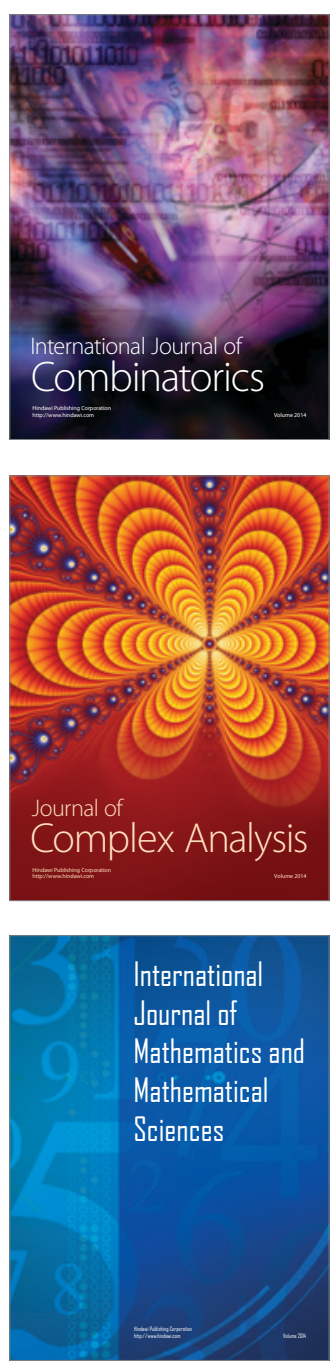
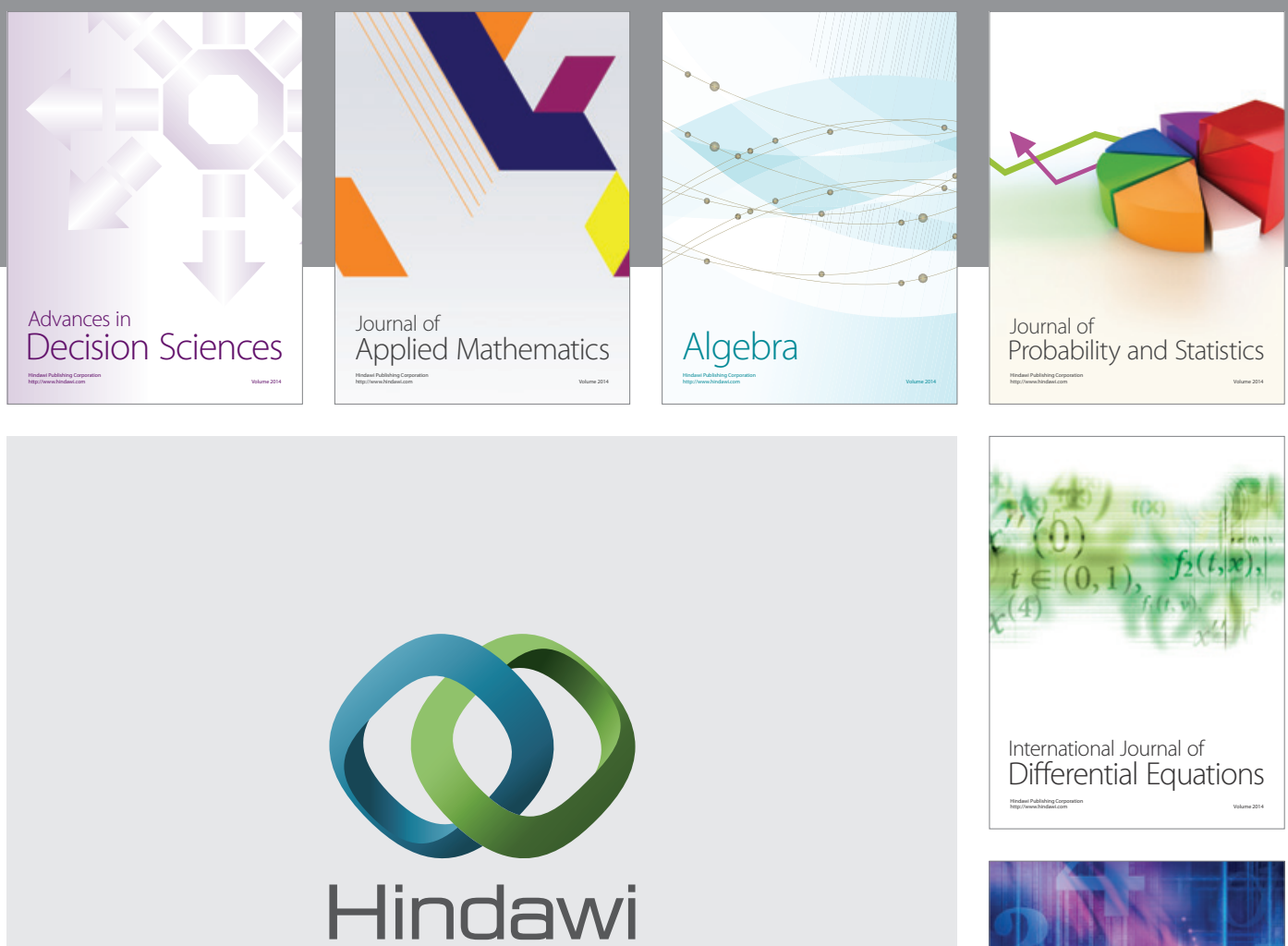

Submit your manuscripts at http://www.hindawi.com
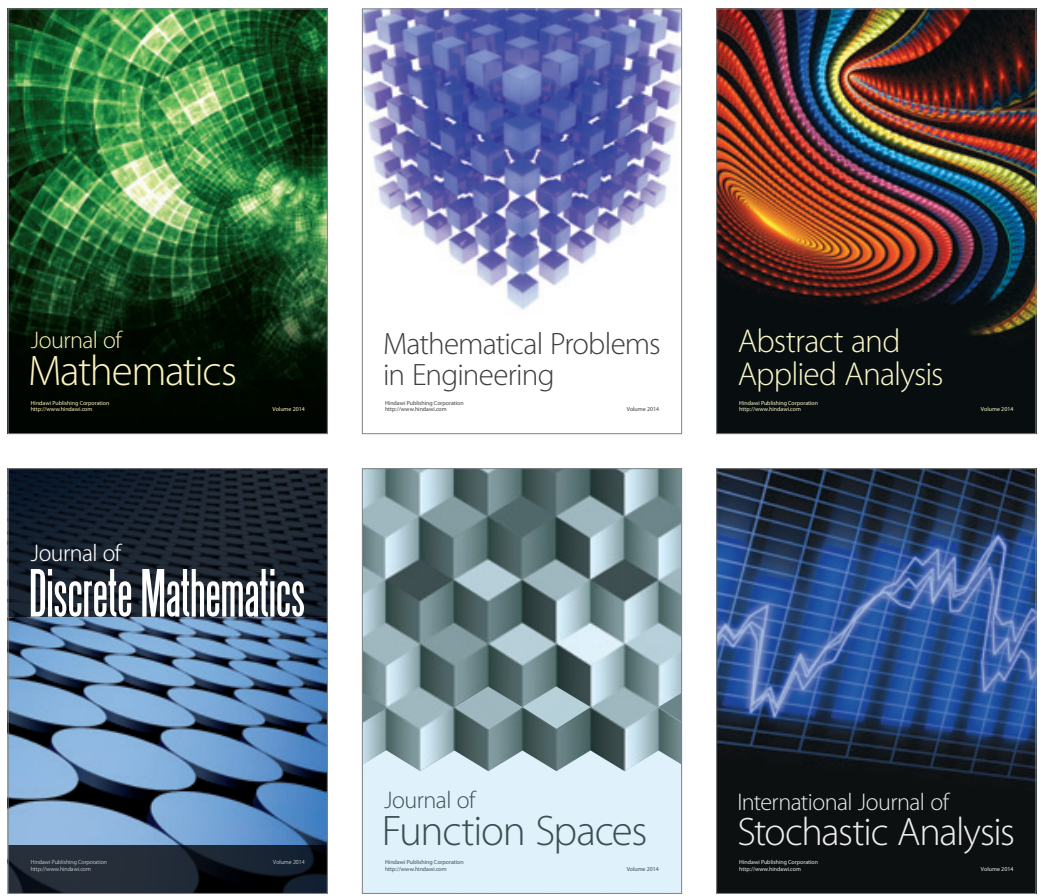

Journal of

Function Spaces

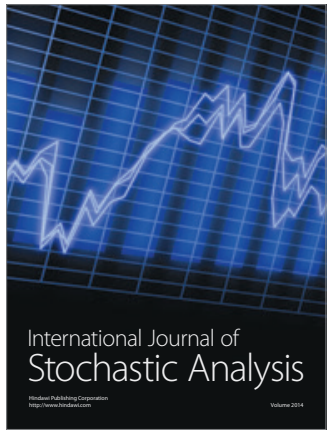

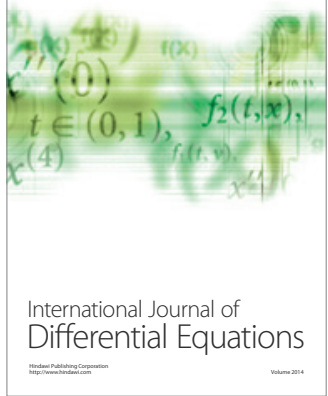
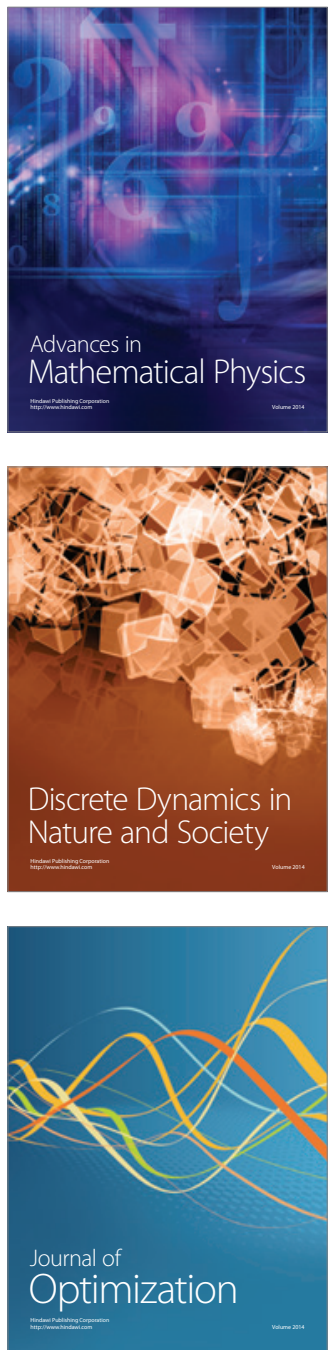